\title{
"Me gustó, porque era una enseñanza viva...": Creencias sobre aprendizaje de lenguas extranjeras de universitarios alemanes que aprenden español como lengua extranjera. Un estudio de caso
}

CARMEN RAMOS

Universität Würzburg

Miquel Llobera

Universitat de Barcelona

Recibido: 12 febrero 2009 / Aceptado 11 junio 2009

ISSN: 1697-7467

RESUMEN: En este artículo, parte de una investigación más amplia, se estudia el pensamiento de una universitaria alemana que estudia formación de profesores de lenguas (francés) y que asiste a un curso intensivo de español como lengua extranjera (E/LE). La investigación muestra la dicotomía que surge en su pensamiento entre la «enseñanza viva» de su curso de E/LE, basada en un aprendizaje activo, dinámico y comunicativo, y sus estudios de formación de profesores, en los que prima la reflexión formal sobre la lengua y la traducción. La alumna acaba cuestionándose sus estudios $\mathrm{y}$, por tanto, su futuro como profesora de lenguas en educación secundaria.

Palabras clave: Pensamiento de aprendices, creencias de aprendices, conocimiento metacognitivo, investigación cualitativa, didáctica de lenguas

"I liked it, because her teaching was lively..." Beliefs of German students of Spanish on language learning. A case study.

ABSTRACT: This paper, as a part of a wider research, studies a German university student's ideating about foreign language learning. She is taking a degree to become a French teacher and she is also attending an intensive Spanish course. The investigation shows a remarkable dichotomy in her thinking between «lively teaching», as experienced in her Spanish course, based on active, dynamic and communicative learning, and her studies in teacher training, with a strong focus on formal linguistic reflection and translation. As a result, she begins to question her university studies and, furthermore, her future as a secondary school language teacher.

Key words: Learners' thinking, learners' beliefs, metacognitive knowledge, qualitative research, language teaching 


\section{INTRODUCCIÓN}

En las dos últimas décadas se ha pasado a considerar al alumno como el elemento central del proceso de enseñanza y aprendizaje. El aprendiz ya no es considerado como un recipiente pasivo de información, sino como miembro activo del proceso de enseñanza y aprendizaje. El aprendiz adulto tiene además una experiencia y un conocimiento del mundo que contribuyen a modelar su forma de aprender, tanto en relación con su estilo cognitivo como con su pensamiento estratégico. Las creencias de un alumno actúan como un filtro a partir del cual percibe y evalúa todo el proceso de aprendizaje.

Las creencias constituyen el fondo de lo que un aprendiz considera que es aprender. Para él, son ideas que se identifican plenamente con la realidad; está convencido de que son verdad. Podemos considerar que las creencias no sólo forman parte de la identidad de un aprendiz de lenguas como tal, sino que contribuyen decisivamente a modelarla.

El presente artículo tiene como objetivo presentar la descripción de las creencias de una universitaria alemana que aprende español como lengua extranjera (E/LE). Esta descripción forma parte de una investigación más amplia que incluye un número mayor de alumnos, así como el estudio de las creencias de un grupo de profesores de educación secundaria, con objeto de comprobar las posibles correlaciones entre uno y otro grupo (Ramos Méndez, 2005).

\section{MarCo teórico}

\subsection{Creencias de los alumnos}

En los últimos años se han realizado algunos estudios interesantes sobre las creencias de los alumnos en torno a cómo se aprende una lengua. Kern (1995) coteja las creencias de un grupo de alumnos con las de sus profesores para comprobar la posible influencia de las creencias de los segundos en las de los alumnos, Mantle-Bromley (1995) relaciona creencias y actitudes de alumnos, Riley (1997) se plantea hasta qué punto las creencias que un aprendiz tiene sobre la lengua influyen en su proceso de aprendizaje y Tumposky (1991) intenta esclarecer hasta qué punto influye el ámbito cultural al que pertenecen los alumnos en sus creencias. Ésta es sólo una muestra de la variedad de temas y variables que se han tratado en torno a las creencias. A ello se añade la variedad de perspectivas de investigación reflejada en Bernat y Gvozdenko (2005) o en Kalaja y Ferreira Barcelos (2006).

Las creencias de un alumno constituyen la base de los juicios y prejuicios con los que un aprendiz aborda el aprendizaje de una lengua. La cuestión central no trata de la veracidad de estas creencias, sino que consiste en saber más sobre ellas, puesto que tienen una influencia notable en el proceso de aprendizaje, ya que: «What they [learners] believe will influence their learning much, much more than what we [teachers, researchers] believe, because it is their beliefs that hold sway over their motivations, attitudes and learning procedures.» (Riley, 1997: 128).

Una de las dificultades que surgen al abordar el estudio de las creencias tiene que ver con la conceptualización de este término, que muchas veces aparece «disfrazado» en forma de múltiples denominaciones. Pajares (1992) menciona, sólo a título de ejemplo, veintiún términos 
diferentes que ha encontrado en la bibliografía. Este autor se marca como objetivo desbrozar el campo conceptual de las creencias, que él llama «messy construct», y ofrece una excelente revisión de este concepto, de sus definiciones, de su relación con el conocimiento, de su naturaleza y características, proponiendo sugerencias para la futura investigación. Pajares concreta la dificultad de conceptualización que surge en este ámbito de esta forma: "Conceptualizing a belief system involves the understanding that this system is composed of beliefs connected to one another and to other cognitivelaffective structures, complex and intrincate though these connections may be, that form beliefs about constructs.» (Pajares, 1992: 316).

Benson y Lor (1999) consideran que las creencias no se dan en todas las circunstancias, sino que son recursos cognitivos para abordar contenidos y contextos de aprendizaje. Por ello, proponen un marco analítico basado en tres niveles: concepciones (conceptions), creencias (beliefs) y maneras de abordar el aprendizaje (approaches to learning). Las concepciones se encuentran en un nivel máximo de abstracción y las maneras de abordar el aprendizaje en el nivel de mayor concreción. Este marco analítico se puede visualizar así:

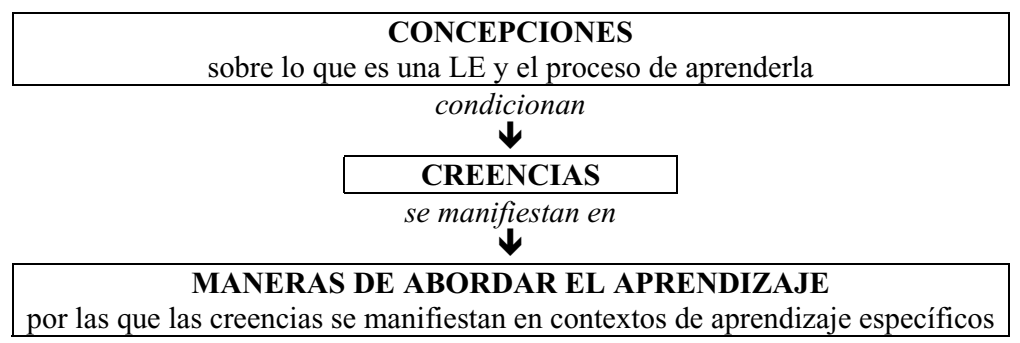

Las «concepciones» nos ayudan a clasificar las creencias jerárquicamente y a agruparlas, mientras que las «maneras de abordar el aprendizaje» nos permiten entender la funcionalidad de concepciones y creencias en un contexto de aprendizaje determinado y la posibilidad de que cambien. El concepto de funcionalidad es el que, en definitiva, determina si la manera de abordar el aprendizaje que tiene un alumno se ajusta en mayor o menor medida al contexto de aprendizaje en el que está inmerso.

\section{Pregunta de investigación}

De forma coherente con el enfoque de investigación etnográfica por el que optamos, no elaboramos hipótesis de investigación, que supondrían la existencia de categorías previamente establecidas. Dado que nuestro objetivo era identificar y describir las categorías que fueran emergiendo de los datos a lo largo del proceso de investigación, formulamos la siguiente pregunta de investigación general:

¿Cómo se articula el pensamiento de adultos aprendices de una lengua extranjera inmersos en una situación de cambio pedagógico? 


\section{Metodología de la investigación}

Las características del tema planteado nos llevaron a decidirnos por un paradigma de investigación etnográfico. Las creencias de los alumnos no son investigables con procedimientos experimentales o casi experimentales, ni medibles por procedimientos psicométricos, sino que podemos intentar descubrirlas, describirlas, explicarlas y relacionar unas con otras, tratando así de trazar una especie de mapa introspectivo de algunos ámbitos del proceso de aprendizaje de un grupo de alumnos.

Para entenderlos necesitamos una aproximación más cercana a la llamada psicología cultural que desarrolla Bruner en sus obras sucesivas In Search of Mind. Essays in Autobiography (1983), Acts of Meaning (1990) y The Culture of Education (1996). Bruner reclama el valor de los relatos autobiográficos y los datos subjetivos para llegar a construir, mediante una acción interpretativa, un conocimiento científico sobre el aprendizaje y el funcionamiento de la mente.

Dicho enfoque se caracteriza por intentar comprender e interpretar los datos obtenidos desde la perspectiva de los participantes en la investigación, es decir, desde la perspectiva émica (Watson-Gegeo, 1988), y abarca no sólo los datos que se refieren a unos conceptos determinados, sino que ha de incluir datos subjetivos, puesto que los hablantes trazan generalizaciones durante la elaboración de su etnografía, ya que se trata de un «acto interpretativo» (Duranti, 2000: 139).

Consideramos el enfoque etnográfico como una de las formas de la investigación cualitativa que atañe a todas las fases del proceso, desde la recogida de datos hasta su interpretación. Van Lier (1988) justifica su defensa de la etnografía en educación por considerar que nuestro conocimiento actual de lo que pasa en las aulas es muy limitado y que habría que aumentarlo. Sin embargo, esto sólo se puede conseguir entrando en las aulas para recoger datos, que, además, han de ser interpretados en el propio contexto del aula, un contexto que no sólo es lingüístico o cognitivo, sino esencialmente social.

Para aclarar el uso que hacemos del término «etnografía», ofrecemos una definición que ya se ha convertido en clásica y que es útil en el marco de este estudio: "Ethnography is the study of people's behavior in naturally occurring, ongoing settings, with a focus on the cultural interpretation of behavior.» (Watson-Gegeo, 1988: 576). Esta idea de la interpretación cultural no es nueva en la tradición etnográfica. Geertz, que publicó en 1973 su conocida obra La interpretación de las culturas, tituló su primer capítulo «Interpretación densa: hacia una teoría interpretativa de la cultura», dando una cierta popularidad en el campo etnográfico al concepto acuñado por Gilbert Ryle, afirmó:

La descripción etnográfica presenta tres rasgos característicos: es interpretativa, lo que interpreta es el flujo del discurso social y la interpretación consiste en tratar de rescatar 'lo dicho' en ese discurso de sus ocasiones perecederas y fijarlo en términos susceptibles de consulta. (Geertz, 1973: 32).

La etnografía estudia una cultura (por ejemplo, el aula) o un grupo dentro de un contexto natural, no experimental. El investigador es un observador atento, pero no manipula. Su interés se centra en obtener los datos dentro del contexto en que se producen. Su finalidad es entender esa cultura o ese grupo, para lo cual realiza un análisis interpretativo y descriptivo 
de los datos, a partir del cual va emergiendo la teoría en forma de códigos y categorías. La presentación de los datos se caracteriza por ser una descripción densa (Geertz, 1973; WatsonGegeo, 1988), por mantener una perspectiva émica, «desde dentro», es decir, desde el punto de vista de los participantes (en contraposición a la perspectiva ética, que usa marcos, conceptos y categorías ya establecidos) y por respetar el principio holístico, que intenta captar el contexto como un todo integral en lugar de seccionarlo en partes. La investigación etnográfica siempre ha de ser una investigación contextualizada.

La etnografía posee un valor social más amplio que el de ser un mero método de investigación. En palabras de Geertz: "[...] el hombre es un animal inserto en tramas de significación que él mismo ha tejido, considero que la cultura es esa urdidumbre y que el análisis de esta cultura ha de ser por lo tanto, no una ciencia experimental en busca de leyes, sino una ciencia interpretativa en busca de significaciones.»(Geertz 1973: 20).

Los datos presentados en este trabajo son parte de una investigación más amplia, la recogida de datos se ha hecho de manera no parcializada ni condicionada y estos han sido objeto de una análisis tan exhaustivo como ha sido posible para poder construir una cierta interpretación que nos permite comprender las concepciones que tiene el grupo de alumnos investigados ante una experiencia de aprendizaje en la cual se presentan elementos inéditos para ellos.

Este tipo de investigación asume el valor de las narraciones personales como fuente de conocimiento (Bruner, 1983) y de las maneras de decir espontáneas como datos significativos para la investigación. A las preguntas sobre la cientificidad de este tipo de investigación que no se basa en estudios de causa-efecto ni en datos que cobran sentido a partir de un estudio estadístico, se puede responder, siguiendo a Geertz:

The ousider view of anthropology as a powerful regenerative force in social sciences and humane studies [...] is an indication that the anthropological way of looking at things', as well as 'the anthropological way of finding things out' and 'the anthropological way of writing about things' do have something to offer the late twentieth century. (Geertz, 2000: 97).

\section{Diseño de la inVestigación}

Los datos del presente estudio se recogieron en el departamento de español como lengua extranjera (E/LE) del Centro de Lenguas de una universidad alemana. Este centro es una institución que ofrece cursos de idiomas a todos los estudiantes de la universidad. En cuanto al enfoque metodológico empleado en las clases de E/LE, es importante señalar que en este departamento se trabaja con un manual que se inscribe en el concepto de enseñanza mediante tareas.

El corpus de datos recogido para el presente estudio es el siguiente:

- Diarios de aprendizaje de 19 alumnos principiantes.

- Fichas con datos personales de los alumnos.

- Entrevistas semiestructuradas con 13 de los 19 alumnos.

- Versión adaptada del cuestionario BALLI (Beliefs About Language Learning Inventory, Horwitz, 1987) de los 19 alumnos como fuente de datos complementaria. 
- Entrevistas etnográficas con 8 profesores de enseñanza secundaria.

- Fichas con datos personales de los 8 profesores.

- Entrevistas etnográficas con las 2 profesoras universitarias de los alumnos.

Partiendo de este corpus de datos se decidió analizar los de 7 alumnas, es decir, sus diarios de aprendizaje y sus entrevistas, así como las entrevistas de 3 profesores de enseñanza secundaria. La selección de las alumnas cuyos datos se tratan en la investigación se hizo atendiendo a la mayor variedad de formación y edad posible. Sobre las razones de que sean sólo alumnas, véase Ramos Méndez (2005). En el caso de los profesores la selección se hizo procurando que estuvieran representados segmentos diferentes en cuanto a la edad, a la experiencia profesional y a las materias impartidas.

La recogida de datos comenzó con los diarios de los alumnos, en base a los cuales se hicieron las entrevistas con ellos. Son estos datos en los que nos centramos en este artículo.

Un estudio de diario es «a first-person account of a language learning or teaching experience, documented through regular, candid entries in a personal journal and then analyzed for recurring patterns or salient events.» (Bailey, 1990: 215). El diario es un método introspectivo, que puede aportar información sobre procesos internos de la persona a los que de otra forma no tendríamos acceso. Bailey (1990) y Díaz Martínez (1998) ofrecen una revisión de la bibliografía sobre los estudios de diarios y los resultados que aportan.

Un principio fundamental a la hora de escribir un diario es que las anotaciones sean sinceras (candid, en palabras de Bailey) y privadas del autor. El procedimiento más frecuente es, a partir de la versión «privada» del diario preparar una versión «pública» del mismo, que es la que se analiza.

Un diario debería incluir informaciones sobre: las razones por las que se escribe, el contexto de aprendizaje o de enseñanza, el profesor y los compañeros de clase, el entorno y la personalidad del autor, el propio proceso de escribir el diario y de darle la forma final, así como la frecuencia y longitud de las anotaciones. Si reúne estos criterios, el diario tendrá significado funcional, que nos permitirá juzgar este tipo de informe individual (Bailey y Ochsner, 1983).

Sin embargo, el problema más importante sigue centrándose en el valor de este instrumento de investigación (Bailey y Ochsner, 1983). Con el fin de disponer de una intersección de diferentes perspectivas, se estudió siempre el diario de cada alumno con la entrevista correspondiente.

Cada alumno entregó la versión pública de su diario (una copia) y se quedó con el documento original. Para el análisis de los diarios, la investigadora los leyó una y otra vez, anotando sucesivamente y refinando las categorías temáticas que iban surgiendo. Al final quedó una lista de 16 categorías temáticas que trataban los alumnos. Las categorías que emergen son: actividades en el aula, experiencia de aprendizaje en el aula y motivación y ansiedad, experiencias anteriores de aprendizaje de lenguas, progresos hechos y estadio del propio proceso de aprendizaje, estrategias de aprendizaje y cómo se aprende, trabajo individual y autoaprendizaje, el factor humano (formas de organización social en el aula, percepción del papel del profesor, percepción y valoración de la interacción por alumnos y alumnas), contenidos culturales, gramática, vocabulario, fonética y pronunciación, destrezas receptivas (comprensión auditiva y comprensión lectora), interacción oral, expresión escrita, uso de material y medios y diario de aprendizaje. 
En un paso posterior, la investigadora categorizó los diarios de los alumnos según estos temas, es decir, elaboró un cuadro sinóptico de cada diario en el que todas las entradas o fragmentos de las mismas se asociaban en torno a cada tema. Así quedaron preparados los diarios para su triangulación con las entrevistas posteriores.

De acuerdo con la metodología cualitativa por la que nos habíamos decidido, realizamos entrevistas etnográficas semiestructuradas para profundizar en los sistemas de creencias, tanto de alumnos como de profesores.

La entrevista etnográfica se trata de una situación comunicativa participativa e igualitaria en la que el entrevistador y el entrevistado dan cuerpo a un proceso de construcción de conocimiento (Kvale, 1996). Se trata de una conversación basada en la participación y con un propósito determinado (Burguess, 1988). Por eso, es importante que el entrevistador establezca una relación de confianza con el entrevistado, que dé pie a la interacción y que permita que este, a través de su discurso, vaya dándole forma a su pensamiento.

En el marco de nuestro estudio, para realizar las entrevistas seguimos las pautas y consejos prácticos que aparecen en la bibliografía (Duranti, 1997; Spradley, 1979; van Lier, 1988), especialmente los descritos por Cohen y Manion (1989) en cuanto a preparación y procedimiento. Se realizaron entrevistas con una guía de preguntas abiertas para facilitar al máximo la interacción. Todas las entrevistas se hicieron en alemán, la lengua materna de todos los entrevistados y en la que estos también habían escrito sus diarios. Posteriormente se tradujeron extractos de las mismas. El análisis se hizo en base a la transcripción del texto original en alemán, respetando los rasgos discursivos de esta lengua.

Tomando como punto de partida los 16 temas que emergieron de los diarios, se confeccionaron preguntas con el fin de que los alumnos pudieran especificar, ampliar y matizar dichos temas. Para todos los alumnos se utilizó la misma guía, pero además, para cada uno de ellos se formularon de una a tres preguntas específicas referidas a algún aspecto concreto de su diario. Todas las entrevistas se transcribieron minuciosamente, incluyendo rasgos discursivos y paraverbales, como pausas, vacilaciones, falsos arranques, interrupciones, alargamientos de sílabas, énfasis, entonación, cambios perceptibles de volumen, solapamientos en la interacción, risas, etc.

Dado que el propósito fundamental del estudio es describir las creencias de alumnos, se utilizan los datos procedentes de las entrevistas con profesores de educación secundaria sólo como datos complementarios que posiblemente nos ayuden a comprender mejor el trasfondo del pensamiento de los alumnos. Por tanto, esta triangulación consiste en un rastreo de unidades temáticas que emergen de los datos de profesores y alumnos, cotejando el contenido de lo que dicen unos y otros y su posicionamiento ante los temas, extrayendo las conclusiones pertinentes y poniendo especial atención a aquellas ocasiones en las que los alumnos, en sus diarios y entrevistas, aluden a su experiencia escolar de aprendizaje de lenguas extranjeras.

Para consultar más detalles de procedimientos de investigación véase Ramos Méndez (2005).

\section{Análisis de datos}

A modo ilustrativo, ofrecemos en este apartado los resultados del análisis de los datos de una alumna, cuyo pseudónimo es Cuzco. Las limitaciones obvias de espacio en este artículo 
nos obligan a centrarnos en una alumna, no pudiendo ofrecer datos de las otras seis analizadas. Nos hemos decidido a presentar los datos de Cuzco por ser una alumna que no sólo aprende E/LE, sino que está formándose para ser profesora de enseñanza secundaria de lengua alemana y de francés. Cuzco hace una reflexión en la que confronta su experiencia actual de aprendizaje de una lengua con sus estudios de preparación como futura profesora. $\mathrm{Su}$ experiencia en el curso de E/LE, en la que se enfrenta a un enfoque metodológico nuevo, la lleva a replantearse su meta profesional.

Incluimos aquí una breve descripción de la alumna y un cuadro con los resultados de la triangulación de los datos surgidos del diario y de la entrevista. En la discusión se destacan los aspectos considerados más relevantes y se añaden algunas reflexiones sobre el pensamiento de esta alumna y su percepción de la enseñanza mediante tareas.

\subsection{Cuzco: descripción de la alumna}

En el momento de recoger los datos, esta alumna tiene 24 años y está cursando sus estudios de Filología (alemán y francés) por dos vías: una para conseguir el título de magister o M.A. y la otra para formarse como profesora de enseñanza secundaria de lengua alemana y de francés, formaciones diferentes en el sistema universitario alemán. Su lengua materna es el alemán. Las lenguas clásicas y extranjeras que estudió en el colegio son: inglés (durante 7 años), latín (también 7 años) y francés (5 años). En la universidad, además del curso intensivo de E/LE en el marco del cual se recogen los datos, sigue también cursos de francés.

Cuzco declara que, en un futuro, le gustaría enseñar a adultos. En varias ocasiones de la entrevista la alumna compara su experiencia como alumna de Filología y como alumna en el curso intensivo de E/LE. A Cuzco le gusta aprender lenguas y está muy motivada para aprender español. Su actitud se puede calificar de entusiasta. En general, Cuzco da la impresión de ser una persona extrovertida y sociable.

Tanto el discurso oral como el escrito de Cuzco la caracterizan como una persona que tiene tacto y que busca formas de expresión que no resulten ofensivas. No obstante, Cuzco es una persona crítica: en varias ocasiones se critica a sí misma o a sus compañeros del curso como aprendices y también hace una crítica abierta de sus estudios de filología.

\subsection{Cuzco: triangulación de datos procedentes del diario y de la entrevista}

En los siguientes cuadros sinópticos se refleja el resultado de la triangulación de datos de Cuzco en referencia con las 16 categorías temáticas analizadas para todas las alumnas (tratamiento general de cada tema), con la percepción que tiene Cuzco de la enseñanza mediante tareas (percepción de cambio metodológico en cuanto a cada tema) y con el contraste entre sus estudios de filología y su aprendizaje de E/LE (tema específico). En los casos en los que las columnas «DIARIO»y «ENTREVISTA» aparecen como una sola hay absoluta coherencia de datos en las dos fuentes. 
Tratamiento general de cada tema

\begin{tabular}{|c|c|c|}
\hline TEMA & DIARIO & ENTREVISTA \\
\hline $\begin{array}{l}\text { 1. Actividades en el } \\
\text { aula. }\end{array}$ & $\begin{array}{l}\text { No le gustan los drills, pero los } \\
\text { considera necesarios al } \\
\text { principio. }\end{array}$ & No aparece este tema. \\
\hline $\begin{array}{l}\text { 2. Experiencia de } \\
\text { aprendizaje en el aula. } \\
\text { Motivación y } \\
\text { ansiedad. }\end{array}$ & \multicolumn{2}{|c|}{$\begin{array}{l}\text { Experiencia de aprendizaje muy positiva basada en: identificación } \\
\text { con la profesora, trabajo en grupos, atmósfera agradable, relación } \\
\text { armoniosa con los otros aprendices, implicación activa de éstos, } \\
\text { variedad de medios. } \\
\text { Escenario de aprendizaje ideal: aprendizaje activo a buen ritmo, } \\
\text { atmósfera distendida y agradable, trabajo cooperativo con } \\
\text { implicación personal, identificación con el profesor y uso de } \\
\text { materiales variados y atractivos. Factor desencadenante de este } \\
\text { escenario: su propia motivación. A mayor grado de dedicación, } \\
\text { mayor motivación. Motivación es esencial para profesor y para } \\
\text { alumno. } \\
\text { Enseñanza preferida: "enseñanza viva", que promueve aprendizaje } \\
\text { activo, dinámico y comunicativo, que fomenta colaboración de los } \\
\text { aprendices en el aula y que requiere implicación, tanto intelectual } \\
\text { como afectiva, de los mismos. } \\
\text { El curso intensivo se ajusta a este escenario de aprendizaje; no así } \\
\text { sus estudios de filología. Su motivación básica es de carácter } \\
\text { comunicativo. }\end{array}$} \\
\hline $\begin{array}{l}\text { 3. Experiencias } \\
\text { anteriores de } \\
\text { aprendizaje de } \\
\text { lenguas. }\end{array}$ & No aparece este tema. & $\begin{array}{l}\text { Innovación didáctica escolar } \\
\text { hacia una enseñanza que } \\
\text { promueva un aprendizaje activo, } \\
\text { comunicativo y cooperativo está } \\
\text { sólo en sus inicios y depende } \\
\text { siempre del profesor. }\end{array}$ \\
\hline $\begin{array}{l}\text { 4. Progresos hechos y } \\
\text { estadio del propio } \\
\text { proceso de } \\
\text { aprendizaje. }\end{array}$ & $\begin{array}{l}\text { Curso intensivo es una base, } \\
\text { pero Cuzco es consciente de } \\
\text { que sólo es un punto inicial de } \\
\text { su proceso de aprendizaje de } \\
\text { español, en el que considera } \\
\text { importante su trabajo } \\
\text { individual. Poder comunicarse } \\
\text { a nivel básico la motiva. }\end{array}$ & $\begin{array}{l}\text { A Cuzco no le gusta hacer } \\
\text { actividades de autoevaluación; } \\
\text { no está acostumbrada a ellas ni } \\
\text { les encuentra utilidad clara (ver } \\
\text { PERCEPCIÓN DEL CAMBIO } \\
\text { METODOLÓGICO: Actividades } \\
\text { en el aula). }\end{array}$ \\
\hline $\begin{array}{l}\text {. Estrategias de } \\
\text { aprendizaje. Cómo se } \\
\text { aprende. }\end{array}$ & $\begin{array}{l}\text { Su aprendizaje es favorecido } \\
\text { por el uso de diversos medios y } \\
\text { materiales. No le da } \\
\text { importancia a la traducción, } \\
\text { porque lo que ella considera } \\
\text { importante es "usar la lengua". } \\
\text { Estrategias de aprendizaje } \\
\text { suyas: aprender vocabulario } \\
\text { con fichas y comprensión }\end{array}$ & $\begin{array}{l}\text { Hay una dicotomía entre la } \\
\text { forma de aprender una LE en sus } \\
\text { estudios de filología (reflexión } \\
\text { formal de la lengua) y el curso } \\
\text { de E/LE (enseñanza } \\
\text { comunicativa). Ella prefiere } \\
\text { claramente esta última. Duda } \\
\text { sobre si dedicarse a la enseñanza, } \\
\text { porque ve dicotomía: enseñar en }\end{array}$ \\
\hline
\end{tabular}


Tratamiento general de cada tema (Cont.)

\begin{tabular}{|c|c|c|}
\hline & $\begin{array}{l}\text { lectora contextualizada y } \\
\text { global. No es suficiente con } \\
\text { comprender un texto; éste es } \\
\text { también fuente de información } \\
\text { sobre la lengua. }\end{array}$ & $\begin{array}{l}\text { la escuela versus hacer algo con } \\
\text { la lengua. } \\
\text { Para aprender una LE es } \\
\text { importante la clase y el profesor. } \\
\text { Cuzco prefiere actividades } \\
\text { significativas, que fomenten el } \\
\text { aprendizaje cooperativo y la } \\
\text { implicación personal, que } \\
\text { empleen material auténtico y que } \\
\text { simulen situaciones reales. } \\
\text { Actividades inefectivas: tipo } \\
\text { cloze o tipo drill. } \\
\text { EMT se adecúa al estilo de } \\
\text { aprendizaje y al concepto de } \\
\text { lengua y de qué es aprender una } \\
\text { lengua de Cuzco. } \\
\text { Es partidaria del aprendizaje de } \\
\text { la LE en situaciones reales de } \\
\text { comunicación. Prefiere aprender } \\
\text { una LE en el país donde se hable } \\
\text { y no en un contexto } \\
\text { instruccional. }\end{array}$ \\
\hline $\begin{array}{l}\text { 6. Trabajo individual y } \\
\text { autoaprendizaje. }\end{array}$ & \multicolumn{2}{|c|}{$\begin{array}{l}\text { Cuzco sabe que una parte importante del aprendizaje de LE es el } \\
\text { trabajo individual, y siente ansiedad cuando no puede hacer lo que } \\
\text { ella considera suficiente. Lo importante si se realiza } \\
\text { autoaprendizaje es la continuidad del trabajo individual. } \\
\text { Aporta entusiasmo por la lengua (también se lo pide al profesor) y } \\
\text { una disposición a hacer trabajos pesados, pero necesarios y a } \\
\text { cooperar con los compañeros. }\end{array}$} \\
\hline $\begin{array}{l}\text { 7. El factor humano: } \\
\text { formas de } \\
\text { organización social en } \\
\text { el aula, percepción del } \\
\text { papel del profesor, } \\
\text { percepción y } \\
\text { valoración de la } \\
\text { interacción por } \\
\text { alumnos y alumnas. }\end{array}$ & \multicolumn{2}{|c|}{$\begin{array}{l}\text { Cuzco tiene una opinión muy positiva sobre el trabajo en parejas y } \\
\text { en grupos: la interacción oral facilita el aprendizaje de recursos de } \\
\text { lengua, hace aumentar la motivación y la participación activa en el } \\
\text { aula, y ayuda a superar la inhibición. Cree que con este trabajo se } \\
\text { aprende porque se pueden simular situaciones reales de } \\
\text { comunicación. Para que sea un trabajo efectivo es preciso que los } \\
\text { otros aprendices se impliquen realmente y que el profesor } \\
\text { compruebe que el objetivo marcado se cumple. } \\
\text { Cuzco se identifica con la profesora del curso. Siente ansiedad } \\
\text { cuando tiene la impresión de que los alumnos (incluida ella) no } \\
\text { cumplen sus expectativas. Se trasluce el hecho de que esta alumna } \\
\text { esté formándose como profesora de secundaria; el grado de } \\
\text { identificación con la profesora es tan alto que a veces incluso se } \\
\text { imagina en su lugar. } \\
\text { La relación humana con profesor/-a le resulta muy importante, }\end{array}$} \\
\hline
\end{tabular}


Tratamiento general de cada tema (Cont.)

\begin{tabular}{|c|c|c|}
\hline & \multicolumn{2}{|c|}{$\begin{array}{l}\text { porque influye directamente en su motivación. A un/-a profesor/-a } \\
\text { le pide entusiasmo por la lengua (que ella también aporta como } \\
\text { alumna), motivación para enseñarla, apertura a la relación con los } \\
\text { alumnos y espontaneidad de reacción. } \\
\text { También le resulta muy importante la buena relación con los } \\
\text { compañeros. Cuzco observa al grupo y es capaz de sacar } \\
\text { conclusiones. Distingue entre relación de los alumnos entre sí y } \\
\text { rendimiento del grupo. Sus preferencias para sentirse bien en el } \\
\text { grupo son: que también los compañeros estén motivados y } \\
\text { dispuestos a trabajar juntos y que el grupo no sea excesivamente } \\
\text { numeroso. }\end{array}$} \\
\hline $\begin{array}{l}\text { 8. Contenidos } \\
\text { culturales. }\end{array}$ & $\begin{array}{l}\text { Cuzco tiene especial interés } \\
\text { por temas culturales sobre } \\
\text { Latinoamérica (su profesora es } \\
\text { uruguaya). }\end{array}$ & No aparece este tema. \\
\hline 9. Gramática. & \multicolumn{2}{|c|}{$\begin{array}{l}\text { Para Cuzco la gramática es una herramienta para mejorar la } \\
\text { comunicación, es un sistema base (lengua como mecano) que sirve } \\
\text { para poder aplicarlo a casos de la comunicación real. En clase hay } \\
\text { que hacer trabajo gramatical (explicaciones por el profesor e } \\
\text { interacción con los alumnos sobre temas gramaticales), pero éste } \\
\text { no debe ser el centro. Los ejercicios de práctica y consolidación de } \\
\text { estructuras gramaticales son trabajo individual. Lo importante no } \\
\text { es saber cosas sobre la lengua, sino saber aplicarlas. Lo esencial es } \\
\text { hablar. } \\
\text { Dicotomía entre su forma de aprender, cuyo objetivo fundamental } \\
\text { es la comunicación, con sus estudios de filología, que dan peso a } \\
\text { la reflexión gramatical y no a su aplicación en situaciones } \\
\text { comunicativas. Esto le produce ansiedad. }\end{array}$} \\
\hline 10. Vocabulario. & \multicolumn{2}{|c|}{$\begin{array}{l}\text { Si se incrementa la cantidad de vocabulario trabajado en clase, } \\
\text { aumenta su motivación. Dicotomía entre estudios de filología y } \\
\text { curso de E/LE. En los primeros, aplica estrategias de aprendizaje } \\
\text { de vocabulario lineales y basadas en la memorización a base de } \\
\text { repetición. En el segundo prueba alguna estrategia nueva, pero } \\
\text { "recae" en la vieja (ver apartado sobre percepción del cambio } \\
\text { metodológico). } \\
\text { Para Cuzco, el vocabulario es crucial para la comunicación; la } \\
\text { gramática, no. }\end{array}$} \\
\hline & No aparece este tema. & No aparece este tema. \\
\hline $\begin{array}{l}\text { 12. Destrezas } \\
\text { receptivas: } \\
\text { comprensión auditiva } \\
\text { y comprensión lectora. }\end{array}$ & \multicolumn{2}{|c|}{$\begin{array}{l}\text { Comprensión auditiva es importante y motivadora para Cuzco. } \\
\text { Para que sea efectiva, la velocidad del habla en las grabaciones } \\
\text { debe ser cercana a la del habla auténtica. Percibe un cambio en los } \\
\text { materiales auditivos: antes la velocidad del habla era más lenta. }\end{array}$} \\
\hline
\end{tabular}


Tratamiento general de cada tema (Cont.)

\begin{tabular}{|c|c|}
\hline & $\begin{array}{l}\text { Una estrategia auditiva importante es "filtrar" la información } \\
\text { relevante. } \\
\text { Todas las destrezas son importantes para la comunicación, pero } \\
\text { prioriza las destrezas orales (hablar y escuchar) frente a las } \\
\text { escritas (leer y escribir). }\end{array}$ \\
\hline 13. Interacción oral. & $\begin{array}{l}\text { Las dos destrezas más importantes para Cuzco son las que } \\
\text { configuran la interacción oral: hablar y escuchar. El pensamiento } \\
\text { de Cuzco en cuanto a la importancia de la comunicación } \\
\text { (especialmente oral), de la interacción con profesor y compañeros, } \\
\text { del trabajo en parejas y en grupos y de la participación activa en el } \\
\text { aula deja claro que la interacción oral es un punto central en el } \\
\text { proceso de aprendizaje de una LE para Cuzco. }\end{array}$ \\
\hline 14. Expresión escrita. & $\begin{array}{l}\text { Cuzco no trata explícitamente } \\
\text { este tema. Escribir es una ayuda } \\
\text { para memorizar, pero también } \\
\text { puede ser comunicarse. }\end{array}$ \\
\hline $\begin{array}{l}\text { 15. Uso de } \\
\text { material/medios. }\end{array}$ & $\begin{array}{l}\text { Cuzco piensa que con variedad de medios y materiales se logra su } \\
\text { ideal de clase: una "enseñanza viva". El estilo de enseñanza de su } \\
\text { profesora le parece muy bueno por ser "multimediático". En } \\
\text { general, su opinión sobre el manual usado es buena, pero le } \\
\text { gustaría contar con índices y glosarios léxicos y gramaticales más } \\
\text { completos que facilitaran su trabajo individual. }\end{array}$ \\
\hline $\begin{array}{l}\text { 16. Diario de } \\
\text { aprendizaje. }\end{array}$ & $\begin{array}{l}\text { Primer diario que escribe Cuzco. } \\
\text { Piensa que sirve para la reflexión } \\
\text { personal y para ser más } \\
\text { consciente de algunas cosas. Es } \\
\text { útil cuando se empieza a } \\
\text { aprender una LE, pero no a nivel } \\
\text { avanzado (dicotomía francés- } \\
\text { E/LE). }\end{array}$ \\
\hline
\end{tabular}

Percepción del cambio metodológico en cuanto a cada tema

\begin{tabular}{|c|c|c|}
\hline TEMA & DIARIO & ENTREVISTA \\
\hline $\begin{array}{l}\text { 1. Actividades en el } \\
\text { aula. }\end{array}$ & \multicolumn{2}{|c|}{$\begin{array}{l}\text { Actividades de autoevaluación: son para ella recapitulación de } \\
\text { contenidos más que reflexión personal. No les ve una utilidad } \\
\text { clara y no está acostumbrada a hacerlas. }\end{array}$} \\
\hline $\begin{array}{l}\text { 2. Experiencia de } \\
\text { aprendizaje en el aula. } \\
\text { Motivación y } \\
\text { ansiedad. }\end{array}$ & No aparece este tema. & $\begin{array}{l}\text { Los datos sugieren que la EMT } \\
\text { se adecúa bien al escenario de } \\
\text { aprendizaje preferido y descrito } \\
\text { por Cuzco como "enseñanza } \\
\text { viva", ya que es para ella un } \\
\text { enfoque metodológico activo, } \\
\text { que: simula situaciones, implica }\end{array}$ \\
\hline
\end{tabular}


Percepción del cambio metodológico en cuanto a cada tema. (Cont.)

\begin{tabular}{|c|c|c|}
\hline & & $\begin{array}{l}\text { un aprendizaje cooperativo, } \\
\text { busca conseguir un objetivo } \\
\text { común, potencia la propia } \\
\text { actividad y el intercambio } \\
\text { comunicativo entre los } \\
\text { aprendices. } \\
\text { Crítica a sus estudios de } \\
\text { filología, porque no se } \\
\text { corresponden con este escenario. }\end{array}$ \\
\hline $\begin{array}{l}\text { 7. El factor humano: } \\
\text { formas de } \\
\text { organización social en } \\
\text { el aula, percepción del } \\
\text { papel del profesor, } \\
\text { percepción y } \\
\text { valoración de la } \\
\text { interacción por } \\
\text { alumnos y alumnas. }\end{array}$ & No aparece este tema. & $\begin{array}{l}\text { Cuzco considera que la EMT } \\
\text { requiere más flexibilidad y } \\
\text { apertura por parte del profesor, } \\
\text { porque es un enfoque } \\
\text { metodológico de procesos y } \\
\text { resultados abiertos. }\end{array}$ \\
\hline 10. Vocabulario. & No aparece este tema. & $\begin{array}{l}\text { Cuzco prueba una estrategia } \\
\text { nueva de aprendizaje de } \\
\text { vocabulario (mapas mentales) y } \\
\text { su opinión es positiva, pero } \\
\text { "recae" en la estrategia de } \\
\text { aprender vocabulario mediante } \\
\text { listas de palabras, repitiéndolas } \\
\text { oralmente y por escrito, porque } \\
\text { le es más eficaz (mejor relación } \\
\text { tiempo-rendimiento). }\end{array}$ \\
\hline
\end{tabular}

Tema específico de la alumna

\begin{tabular}{|l|l|l|}
\hline \multicolumn{1}{|c|}{ TEMA } & \multicolumn{1}{|c|}{ DIARIO } & \multicolumn{1}{c|}{ ENTREVISTA } \\
\hline $\begin{array}{l}\text { Contraste entre sus } \\
\text { estudios de filología } \\
\text { francesa } \\
\text { aprendizaje de E/LE el }\end{array}$ & $\begin{array}{l}\text { Este contraste va surgiendo en los diferentes temas (experiencia de } \\
\text { aprendizaje en el aula, estrategias de aprendizaje, gramática) y es } \\
\text { en esos apartados donde se trata. La diferencia básica es que el } \\
\text { curso de E/LE que ha experimentado representa su ideal de clase: } \\
\text { "enseñanza viva", y los estudios de filología no. }\end{array}$ \\
\hline
\end{tabular}

\subsection{Cuzco: discusión de los datos}

En esta discusión de los datos nos centraremos, por motivos de espacio, en el tema específico de la alumna: el contraste que hace entre sus estudios de filología y su proceso de aprendizaje de E/LE en el curso intensivo. Este tema nos parece interesante, puesto que 
esta alumna reflexiona en un doble plano: como alumna de E/LE, pero también como posible futura profesora. Al estudiar las creencias de esta alumna podemos considerarla como una especie de «puente» entre alumno y profesor.

Destacamos a continuación los dos aspectos de este tema que nos parecen más relevantes: la experiencia de aprendizaje en el aula y la forma de aprender una lengua extranjera. Las citas que combinan la letra $" \mathrm{C} »$ con un número (por ejemplo, C81) hacen referencia a la intervención exacta de la entrevista; las citas que se marcan con una fecha corresponden a la entrada del diario escrita el día correspondiente. Por motivos de espacio ofrecemos la traducción al español de las citas originales en alemán. Estas se pueden consultar en Ramos Méndez (2005).

a) La experiencia de aprendizaje de Cuzco durante el curso intensivo de E/LE es altamente positiva desde el principio hasta el final, por lo que el curso potencia su motivación, que se basa en los siguientes factores: atmósfera agradable y buena relación con compañeros y profesora, ritmo no demasiado lento y adecuado a lo que ella se cree en condiciones de aprender, progresos hechos durante el curso, aprendizaje cooperativo, tratamiento de contenidos culturales sobre Latinoamérica (de donde procede su profesora), trabajo con canciones, el material adicional empleado en el curso y, en general, el manual.

Cuzco emplea una metáfora para describir el tipo de enseñanza que ella considera ideal para aprender una lengua y que al mismo tiempo aplica al curso intensivo de E/LE: enseñanza viva («lebendiger Unterricht», C81). Para Cuzco ésta es una enseñanza que promueve un aprendizaje activo, dinámico y comunicativo, que fomenta la colaboración de los aprendices en el aula y que requiere la implicación, tanto intelectual como afectiva, de los mismos. Su concepto de «enseñanza viva» es plenamente aplicable al curso intensivo de E/LE; no así a sus estudios de filología, con los que se siente bastante decepcionada (C12 y C13). Esta idea queda bien reflejada cuando Cuzco explica cómo es para ella una buena clase de lengua (M. es su profesora de E/LE; las mayúsculas indican énfasis; los signos de ortografía reflejan el flujo discursivo, no la ortografía):

C12: ehm sí cuando yo me DIVERTÍA un poco, creo yo, o sea, eso siempre era así como con M., sencillamente que nos reíamos un poco y que nosotros mismos teníamos que hacer cosas, cuando uno podía implicarse, eso siempre me gustaba UN MONTÓN y cuando uno tenía que formular algo y le salía y- sí- ahí yo no tenía tantos reparos, sí: ehm porque en mis estudios normales ((risas)) yo más bien muchas veces tengo reparos de decir algo y...

b) Donde más patente se hace el contraste que establece Cuzco entre sus estudios de filología y su aprendizaje de E/LE es en el tema dedicado a las estrategias de aprendizaje y a cómo se aprende. En esa comparación, siempre salen perdiendo sus estudios de filología:

Quiero saber usar la lengua y saber expresar y comprender contenidos - traducir es (casi) totalmente superfluo (se ve que estoy realmente harta de mis estudios...)» $(18.09 .00)$

En este fragmento reflexiona sobre la cuestión de si a ella le gustaría cambiar algo o no en su forma de aprender lenguas: 
C9: sí, me gustaría cambiar algo, o sea estoy intentando en francés por ejemplo porque, claro, echo de menos el hablar y en la universidad casi no hablamos nada, no como aquí, ehm por lo menos ahora leo artículos de periódico, porque creo que me resulta útil, también para el vocabulario y ehm ahora me encuentro con unas amigas para ampliar el vocabulario y... yo siempre he sabido que me fío siempre de mi intuición en la gramática y | ehm eso creo que a la larga no es lo mejor para el examen de Estado ((risas)), o sea yo tengo que... un poco de gramática, me tendría que dedicar un poco más a la gramática, creo yo, pero... sí o simplemente hablar más con gente, porque entretanto tengo casi un poco de miedo de hablar con la gente, porque uno se desacostumbra y o sea yo estuve bastante tiempo en Francia, pero cuando uno ya no está acostumbrado, o sea, eso debería cambiarlo, pero por lo menos de momento intento ehm aprender palabras, pero debería volver a hacer gramática y así bien...

E10: sí, sí, ¿hablas ahora más bien con vistas al examen de Estado o, en general, con vistas a aprender una lengua?

C10: ehm | bueno o sea bien, el objetivo próximo, claro, es el examen de Estado de alguna manera, porque de alguna manera hay que sacarlo y es- pero por otro lado me parece tan absurdo las cosas que preguntan ahí y qué palabras tenemos que saber y que yo nunca más voy a necesitar y que yo en parte no conozco en alemán y eso, claro, es poco motivador y uno se queda con poco, me doy cuenta, pero en principio claro que es la lengua lo que siempre me interesa y con el español sobre todo, porque me parece precioso y me parece bonito entrar en contacto con otras personas y poderse entender. Eso es en realidad... sí... justo por eso no sé si de verdad quiero ehm enseñar en la escuela, sino más bien hacer algo yo misma con la lengua...

Es decir, Cuzco se plantea el dilema: enseñar en la escuela versus hacer algo ella misma con la lengua. Es, pues, comprensible que esta alumna se replantee su futuro profesional como profesora de enseñanza secundaria.

$\mathrm{Si}$, basándonos en los datos, tenemos en cuenta que Cuzco es una alumna motivada, responsable de su aprendizaje, cuyo objetivo primero es la comunicación, dispuesta a implicarse en el aula y con preferencia por el aprendizaje cooperativo, podemos deducir que la enseñanza mediante tareas es un enfoque muy apropiado para ella. En este caso, el nuevo enfoque da en el blanco de las expectativas y creencias que esta alumna tiene en torno a lo que significa aprender una lengua extranjera.

\section{Conclusiones}

En el presente artículo hemos intentado demostrar la importancia que tiene saber más sobre las creencias de los alumnos en torno al aprendizaje de una lengua extranjera. Dichas creencias actúan como un filtro por el que el alumno percibe todo el proceso de aprendizaje; además, son un determinante de las decisiones que toma el alumno al aprender, por ejemplo, a la hora de seleccionar y aplicar estrategias de aprendizaje.

No es fácil delimitar el campo de las creencias, por estar unido a otros factores afectivos y cognitivos como las actitudes y el aprendizaje metacognitivo. Por otra parte, hay factores, 
muchas veces difícilmente acotables, que inciden en las creencias, como la cultura de aprendizaje en la que el alumno está inmerso o sus experiencias anteriores de aprendizaje de lenguas. Por tanto, pensamos que es pertinente estudiar las creencias de los alumnos en relación con las de los profesores, sin que necesariamente se dé entre ellas un fenómeno de causa-efecto.

Si queremos avanzar en el campo de la didáctica de las lenguas extranjeras, no podemos fiarnos de estereotipos de aprendices, sino que necesitamos información más sólida, fundamentada en la investigación. La alumna que hemos presentado en este artículo a modo de ejemplo no corresponde al esteretipo vigente en la «cultura popular» sobre los aprendices alemanes. Cuando el enfoque metodológico experimentado en el aula por los alumnos se ajusta a sus creencias y, por ende, a sus expectativas, el proceso de aprendizaje se percibe como más beneficioso. Pero si la profesora de esta alumna se hubiera fiado de los estereotipos vigentes sobre aprendices alemanes, no habría llegado a probar la enseñanza mediante tareas en este contexto.

Más que estudiar las creencias de los alumnos según su procedencia cultural, pensamos que es conveniente investigar más sobre los factores que influyen en dichas creencias y por qué, así como indagar sobre qué efectos concretos tienen las creencias en el proceso de aprendizaje de una lengua extranjera y cuál es su relación con la forma de percibir lo que ocurre en el aula. Éstos son los retos de investigación para un futuro próximo.

\section{Bibliografía}

Bailey, K.M. (1990). "The use of diary studies in teacher education programs", en J.C. Richards y D. Nunan (eds.) Second Language Teacher Education. Cambridge: Cambridge University Press, 215-226.

Bailey, K.M. and Ochsner, R. (1983). "A methodological review of the diary studies: windmill tilting or social science?”, en K.M. Bailey, M.H. Long y S. Peck (eds.) Second Language Acquisition Studies. Rowley (Mass.): Newbury House, 188-198.

Benson, P. y Lor, W. (1999). "Concepcions of language and language learning”, System, 27: 459472.

Bernat, E. y Gvozdenko, I. (2005). "Beliefs about language learning: Current knowledge, pedagogical implications, and new research directions", en TESL-EJ, 9, 1: 1-21.

Bruner, J. (1983). In Search of Mind. Essays in Autobiography. Harper \& Row. New York.

Bruner, J. (1990). Acts of Meaning. Cambridge: Harvard University Press.

Bruner, J. (1996). The Culture of Education. Cambridge: Harvard University Press.

Burgess, R.G. (1988). "Conversations with a purpose: the ethnographic interview in educational research”, en R.G. Burgess (ed.) Studies in Qualitative Methodology. Vol. 1: Conducting Qualitative Research. London/Greenwich (Connect.): Jai Press, 137-155.

Cambra Giné, M. (2003). Une approche ethnographique de la classe de langue, Paris: Didier.

Colás Bravo, M.P. y Buendía Eisman, L. (1994). Investigación educativa. Sevilla: Alfar (2a ed).

Díaz Martínez, J. (1998). Afectividad y tratamiento del error en la clase de segundas lenguas: Estudio introspectivo de un diario. Memoria del Máster de Formación de Profesores de Español como Lengua Extranjera, Universidad de Barcelona (http://www.sgci.mec.es/redele/ biblioteca/diaz.htm). 
Duranti, A. (1997). Antropología lingüística (trad. P. Tena, 2000). Madrid: Cambridge University Press.

Ellis, R. (2003). Task-based Language Learning and Teaching, Oxford: Oxford University Press.

Freeman, D. (1992). "Language teacher education, emerging discourse, and change in classroom practice", en J. Flowerdew, M. Brock y S. Hsia (eds.) Perspectives on Second Language Teacher Education. Hong-Kong: City Polytechnic of Hong Kong, 1-21.

Geertz, C. (1973). La interpretación de las culturas (trad. A.L. Bixio, 1997). Barcelona: Gedisa.

Geertz, C. (2000). Available Light. Princeton: Princeton University Press.

Gil, X., García, E. y Rodríguez, G. (1995). "Anàlisi de dades en la investigació etnogràfica”, en Temps d'Educació, 14: 61-82.

Holliday, A. (1996). Appropriate Methodology and Social Context. Cambridge: Cambridge University Press.

Horwitz, E.K. (1987). "Surveying student beliefs about language learning", en A. Wenden y J. Rubin (eds.) Learner Strategies in Language Learning. Englewood Cliffs: Prentice-Hall, 119-129.

Horwitz, E.K. (1999). "Cultural and situational influences on foreign language learners' beliefs about language learning: a review of BALLI studies", en System, 27: 557-576.

Kalaja, P. y Ferreira Barcelos, A.M. (eds.) (2006) Beliefs about SLA: New Research Approaches, New York: Springer.

Kern, R.G. (1995). “Students' and teachers' beliefs about language learning”, en Foreign Language Annals, 28, 1: 71-92.

Kvale, S. (1996). Interviews: An Introduction to Qualitative Research Interviewing. Thousand Oaks (CA): Sage.

Mantle-Bromley, C. (1995). "Positive attitudes and realistic beliefs: links to proficiency", en The Modern Language Journal, 79, 3: 372-386.

Nunan, D. (1992). Research Methods in Language Learning. Cambridge: Cambridge University Press.

Pajares, M.F. (1992). "Teachers' beliefs and educational research: cleaning up a messy construct", en Review of Educational Research, 62, 3: 307-332.

Ramos Méndez, C. (2005). Ideaciones de estudiantes universitarios alemanes sobre su proceso de aprendizaje de español como lengua extranjera ante una enseñanza mediante tareas. Tesis doctoral (dirigida por M. Llobera). Universidad de Barcelona (http://www.tdx.cbuc.es/ index_tdx_cs.html).

Reinartz, A. (2003). Leben und Lernen sind weit auseinander! Eine Studie zur Rezeption der handlungsorientierten Didaktik durch Englischlehrerinnen und-lehrer am Gymnasium, Opladen: Leske \& Budrich.

Riley, P. (1997). “BATS' and 'BALLS': beliefs about talk and beliefs about language learning”, en Mélanges CRAPEL, 23, 1: 125-153.

Skehan, P. (2003) "Task-based instruction", en Language Teaching, 36: 1-14.

Tumpovski, N.R. (1991). "Student beliefs about language learning: a cross-cultural study", en Carleton Papers in Applied Language Studies, 8: 50-65.

van Lier, L. (1988). The Classroom and the Language Learner: Ethnography and second-language classroom research. London: Longman.

van Lier, L. (1990). "Ethnography: Bandaid, Bandwagon, or Contraband?”, en C. Brumfit y R. Mitchell (eds.) Research in Language Classroom. London: Modern English Publications \& The British Council, 33-53.

Watson-Gegeo, K.A. (1988). "Ethnography in ESL: Defining the Essentials", en TESOL Quarterly, 22, 4: $575-592$. 
Woods, P. (1992). "Symbolic Interactionism: Theory and Method”, en M.D. LeCompte, W.L. Millroy y J. Preissle (eds.) The Handbook of Qualitative Research in Education. San Diego: Academic Press, 337-404. 